\title{
The perceptual-cognitive foundation of the subject-predicate structure
}

\author{
Author: Kaoru Takamatsu \\ e-mail: ckeyv910@sutv.zaq.ne.jp \\ Affiliation: Unaffiliated \\ Funding: No fund, grant, or other support was received.
}

\begin{abstract}
This article describes a theoretical attempt to found the subject-predicate structure of declarative sentences on a framework of the human perceptual-cognitive system. The basis of this study is the idea that perception and cognition of events in the world would form mental representations in the system, a kind of models of the events that embody pieces of information about the events. This idea suggests that such models have structures that correspond to grammatical structures of the linguistic expressions that represent the events and express the pieces of information embodied by the models. The model structures that correspond to the subject-predicate structure and logical connectives have been constructed within the scope of the computational theory of the system following the way in which the system should function to form the models of the events. This construction of the structures entails propositional logic. Application of the structures to the liar paradox leads to a new solution of this paradox.
\end{abstract}

Keywords: grammatical structures; the human perceptual-cognitive system; mental representations; propositional logic; the liar paradox . 


\section{Introduction}

The perception and cognition $(P C)$ of events is production of pieces of information about (or mental representations of) the events, which typically linguistic expressions express and convey. Since the human perceptual-cognitive system ( $P C S$ ) stocks some of them, these pieces of information must have physical foundations embodying them.

A physical foundation of the piece of information about an event, 'An object $a$ has a property $P$ ', should be something that corresponds to the event via the following stages:

(1) Generation of physical (or chemical) effects from the object and its property on the PCS (e.g., the object causes certain dynamic effects and a property of the object causes, for instance, certain wavelength of light).

(2) Processing in the PCS of the effects to produce the information. Since the properties of an object cannot exist without the object, the effects of the properties are subordinate to that of the object. Thus, the information about an event should consist of components that correspond to the two kinds of effects combined together in the structure that corresponds to this antecedent-subordinate relationship. Accordingly, the physical foundation that embodies the information will have a structure homomorphic to this relationship. Moreover, the components that correspond to the two kinds of effects should also be physical entities homomorphic to their effects; in general, the effects have structures determined by structures of objects or properties, e.g., patterns of spatio-temporal ( $s t-)$ distributions, intensity, quality, or quantity.

Thus, these physical foundations are regarded as models of events, objects or properties. In the same way, according to a 
variety combinations of events (e.g., conjunction, implication) or those of objects and properties, there should exist physical foundations that correspond to these combinations. Therefore, I raise a hypothesis that the PCS forms physical foundations that are homomorphic to events, objects, properties and their combinations. I call these physical foundations $i$-models of them.

On the other hand, the linguistic expressions that express the pieces of information about events consist of components that express the components of the pieces of information. The linguistic counterparts of the information components should be combined in structures that correspond to the structures of the information. The basic structures of linguistic expressions that express information are grammatical structures. Therefore, i-models that embody the information should have structures homomorphic to certain grammatical structures. If this is the case, the i-model structures can be regarded as the perceptual-cognitive foundations of the grammatical structures. This poses the problem of what these imodel structures are like. Since these structures should be defined in terms of the PC, the mechanism of the PCS is the precondition for working on this problem.

However, we have not yet made the mechanism of the PCS clear. Even the computational theory (D. Marr 1982. Section 1.2, pp 19-30) of the PCS seems to be undeveloped, that is, the theory of the PCS framework to attain the purpose of the PCS. The purpose of the PCS must be acquisition of information useful for survival of the agents with the PCS (Agent). A typical kind of information for this purpose would be that about regularities of the world. Agent can guess the future or unknown situations using information of the regularities and can act advantageously. This advantage would have caused evolution pressure to form the PCS of living things that has the faculty to acquire information about the regularities. Thus, I try to sketch the computational theory of 
the PCS based on this purpose and work on the problem of the imodel structures within the scope of this computational theory.

This line of research on structures of information or mental representations seems worthy of doing. However, to the best of my knowledge, this constructive strategy seems undeveloped. Some studies focus on the PCS from an evolutionary perspective (Anderson, 1990.pp. 26-37, Geisler and Diehl, 2003). They discuss the evolution of the perceptual system. However, they neither target the acquisition of information in the PCS nor compare the information with grammatical structures.

Langacker (2008, pp.31) focuses on the phenomenological aspect of the PC to investigate grammatical structures, but does not target the functional aspect of the PC: how to acquire information from environmental input or what structures the information should have. Langacker takes the meaning of, or the information expressed by a sentence as an elaboration of the concept of relation expressed by the predicate using the concept expressed by the subject (Langacker, 2008, Sections 3.3, pp. 66-72, and section 11, pp. 354405, 1987. Section 8.4, pp. 310-324). His interpretation of sentences lacks the PC process of the elaboration.

Jackendoff takes the information conveyed by a sentence as the concept of an event or a state obtained from a function: from a mental representation of a thing(s) to a mental representation of an event or state about the thing(s) (Jackendoff, 2002, Section 11.8, pp. 360-368, 1983. Chapter 4.2, pp. 63-70, and Chapter 5.1, pp. 7782). This interpretation also lacks the PC process to form this function and the structure of this function. This PC process and the function structure should be crucial for founding the information expressed by sentences on the PC.

King and Soames discuss the relation between the constituents of propositions expressed by declarative sentences (King, 2019. 2018, pp. 1-40, Soames, 2018, pp. 235-252). They take the relation 
as ascription or predication of the information content or semantic value expressed by the predicate of a sentence to that expressed by the subject. However, no cognitive processing based on which the ascription or predication should take place is given. Without perceptual-cognitive foundations, we cannot objectively understand the 'ascription' or 'predication'.

Thus, I found no reports in the literature that makes an attempt to found grammatical structures on the PCS.

\section{Formation of $i-m o d e l s$}

\subsection{I-models of properties}

Objects in the world have a variety of properties that cause a variety of physical or chemical effects. The effects caused by each of the properties are not random but are classified according to certain relationships, such as similarity between features of the effects. For instance, there exist finite kinds of substances, each of which has a specific property that causes certain effects similar to each other that form a cluster. Then, such a similarity relationship is regular within the cluster, and the effects have a regular pattern: an effect connected by the similarity.

Because of the regularities, the regular patterns of effects can be targets for the PCS to form their i-models. What the PCS perceives are their effects of properties and relationships between them. Thus, the regular pattern of the effects in a cluster is identical with the property for the PCS. Hence, I call the i-models of the regular patterns property models. Then, the PCS should have a faculty to form property models i.e., syntheses homomorphic to the regular patterns, from those effects as input stimuli. To form property models, the PCS must have an operation to form syntheses homomorphic to those features of the effects. The syntheses are 
called input images. Then, the similarity between the features will be homomorphic to a relationship between the input images, and the regular pattern is homomorphic to a certain common pattern of the input images. This common pattern will be the i-model of the regular pattern, i.e., the property model. The operation to extract the common pattern from the input images is denoted by $C_{t}$. Given a distribution of input images, $C_{t}$ would extract a common pattern if it exists, hence also functions as categorization of new input images into the property models acquired previously.

It is plausible that the PCS acquired the faculty to form property models along the course of evolution. Therefore, I postulate that the PCS has the operations stated above.

Because inputs are partial and fragmented, property models are conjectures of the properties. Therefore, evaluation of common patterns in input images by $\mathrm{C}_{t}$ is not necessarily decisive. An input image of an object's property can vary according to the situation to accept the input. Then, the evaluation would be updated. For instance, a beast may be regarded as a horse in the distance. However, you may find it a donkey when you approach it. Thus, a property model, say, $B$ has to take two modes in perception according to whether $B$ is adopted or rejected as the property model of an input image $d$, the result of $\mathrm{C}_{\mathrm{t}}(d)$. (It should be noted that to change the evaluation of input images of a property, the input images must be regarded as those of the property of the same object. When $B$ is adopted the mode of $B$ to $d$ (Mode $(B \mid d))$ is denoted by + , and $B^{+}$denotes the adopted $B$. When $B$ is rejected, Mode $(B \mid d)$ is denoted by - , and $B^{-}$denotes the rejected $B$. To reject $B, B$ must be adopted as a candidate first (i.e. $B^{+}$precedes $B^{-}$). When $C_{t}$ categorizes an input image $d$ into $B, \operatorname{Mode}(B \mid d)=+$ and the property model resulting from $d$ is $B$. When the succeeding perception rejects $B, B^{+}$must be changed into $B^{-}$. The i-model that results from this change is denoted by $B^{n}$, where $\mathrm{N}$ is the operation 
that changes $B^{+}$into $B^{-}$or $B^{-}$into $B^{+}$. Since the results of superposition of Operation $\mathrm{N}$ are reduced to $B^{+}$or $B^{-}$, the property models obtained from inputs result in $B$ or $B^{N}$.

\subsection{I-models of objects}

- Agent model

The effect caused by the existence of an object or object-hood is dynamic one. This effect typically appears when the object has contact with other objects. Then, the effect is within the object. Therefore, for the PCS to form the i-models of objects, direct inputs about object-hood from within the objects into the PCS are necessary. Objects away from Agent provide no such inputs into its PCS. Agent itself provides the input into the PCS; the peripheral nervous system in Agent directly perceives the dynamic effects from the agent body from which the PCS forms input images of Agent. Then, the effect is given st-continuously, and input images are divided into st-values of the st-positions of Agent and each of the input images has its st-value.

Since Agent, as an object, keeps its identity regardless of variation of its st-position, it has regularity. This regularity corresponds to the commonality of the dynamic inputs to form the input images of Agent. Thus, an element that takes mode + when any of the input images is given identifies them, hence, corresponds to Agent via the input images, namely, the i-model of Agent (Agent model denoted by $I$ ). $C_{t}$ should introduce such an element when those input images are presented.

Then, What is the i-model of the situation where Agent is at an st-position? The input image based on the input from the situation is a conjunction of that of Agent and the st-value. Thus, its i-model will be the conjunction of Agent model and the st-value: Both of them take mode + to the input from the situation iff the 
conjunction takes mode + . This conjunction is denoted by I-an stvalue. Then, the i-model of 'Agent at an st-position' is the i-model of Agent whose mode is + under the condition that Mode $(\mathrm{I}-\mathrm{P} \mid d)=$ + , where $\mathrm{P}$ is the st-value of the st-position of Agent and $d$ is the input image. $\mathrm{I}^{*} \mathrm{P}^{*}$ denotes this $\mathrm{i}-\mathrm{model}$. This combination is called r-combination.

Then, Agent at st-positions provides inputs that have the regular pattern that consists of a constant component from Agent and a variable component about st-positions of Agent. The former component corresponds to Agent model and the latter component does to the i-model of an arbitrary st-positon, which is denoted by a variable $x$. Then, the i-model of Agent at an st-positon is $\mathrm{I}^{*} x^{*}$.

Since $x$ is a variable varying through the st-positions of Agent, $I^{*} x^{*}$ is a function from an st-value to the i-model of the Agent at the st-position. The PCS can acquire this function by applying $\mathrm{C}_{t}$ to the series of the r-combinations: comparing the r-combinations to find out constant components and varying components in the rcombinations and eliminating the varying components.

Elimination works in the following way. Let $M$ be a composite that includes a component $u$ at some positions in $M$, say, a formula $u \times b+c-u$. Then, let $E L(u / M)$ denote the operation that eliminates $u$ from $M$, whose result is $\# \times b+c-\#$, where \# is the field to put the element that $\times$ or - connects to $b$ or $c$, respectively. This field $\#$ is necessary, because the converse operation of $\operatorname{EL}(u / M), \operatorname{EL}^{-}(u / M)$, on the result of $\operatorname{EL}(u / M)$ recovers $M$. Thus, the result of $\operatorname{EL}(u / M)$ is not a simple residual of $M, b+c$, but one that includes the connecters, $\times$ or - with the field \# to connect a component in the fields. Naturally, the field in the result of an EL is specific to the EL. Thus, the fields resulting from plural ELs must be discriminated according to the ELs. The discriminated fields indicate to which connecters a component should be assigned when an $\mathrm{EL}^{-}$draws composite from the result of the EL by applying a 
component. For example, when $b$ is a component of $M$ different from $u$, then, $\operatorname{EL}(u / M) \neq \operatorname{EL}(b / M): \#_{1} \times b+c-\#_{1} \neq u \times \#_{2}+c-u$.

Such discriminated fields are represented by variables, which distinguish the fields discriminated by ELs that operate on different components.

$\mathrm{EL}(u / M)=M_{u}(x): x \times b+c-x$.

$\operatorname{EL}(b / M)=M_{b}(y): d \times y+c-d$.

$\operatorname{EL}(b / \operatorname{EL}(u / M))=\operatorname{EL}\left(b / M_{u}(x)\right)=M_{u b}(x, y): x \times y+c-x$.

Then, any element in the domain of an EL can be connected by the $\mathrm{EL}^{-}$to the result of $\operatorname{EL}(u / M)$. That is, $u$ can be replaced by another element, e.g., $g \times b+c-g$, when $g$ is in the domain of the EL.

By applying an EL to the series of Agent models stated above, the PCS acquires the i-model of the regular pattern of the $r$ combinations about $I$, i.e., $I * x *$. The i-model of the relationship between Agent and its property is also conjunction. The domain of $x$ in this function can be extended to property models.

Thus, $I$ is also followed by the i-model of the outward (a property model) of the agent body, which can be formed using, for instance, a visual input. This property model has the st-value that corresponds to the st-position of Agent. The input image of the agent body (as an object) combines with the input image of the agent outward (as a property) at the same st-position. This combination corresponds to the conjunction of $I$ and i-model of the agent outward via their common st-value. The i-model of Agent with its outward is the r-combination of i-models of them.

- Object models

Since the relationship between an object and its outward is parallel to that between Agent and its outward. The object is supposed to exists on the basis of its outward and this parallelism of the relationship. By applying the relationship between Agent 
model and the Agent outward model to the object outward model, the object model can be composed. That is, the object model is the counterpart of Agent model as an extension of the projection:

Agent outward model $\rightarrow$ the object outward model to relationship between Agent model and Agent outward model. The PCS can use the projection and the relationship because they are formed in the PCS.

Existence of the counterpart in the world of an object model is a conjecture based on its outward model. In general, some properties of an object that cause sound or smell with its stposition etc, can also play this role of the object outward.

The i-model of an (conjectured) object specified by the measured value $l$ of its st-locus is denoted by $O^{l} . O^{l}$ is an element in the PCS that would correspond to an object, if the object really exists. Then, the situation that consists of the object and its property whose i-model is, say, $B$ will correspond to $O^{l}-B$. Therefore, the i-model of the object specified by the property $B$ is $O^{l *} B^{*}$. In this r-combination, $B$ specifies a range in $l$, because a property applies to objects in a certain st-range. For instance, the physiological properties of a dog applies to the dog anytime, diet of a dog applies to a behavior of the dog that takes for about thirty seconds, or the longevity of a dog applies to its entire life. Therefore, a property model combines with the object model with the st-value of the st-range to which the property applies.

The PCS should also form i-models of categories of objects. A typical category of objects is the set of the objects satisfying a certain regularity by which the objects form a cluster, such as species of living things, chemical substances or artifacts. Thus, the i-model of such a category of objects (a category model) is the imodel of objects with a regularity. This category model is a function, $O^{x *} A^{*}$, where $A$ is the i-model of the regularity (a property model) and $x$ is the variable ranging over objects' loci. 
That is, $\mathrm{O}^{x}$ is the variable ranging over objects.

An object category can be restricted by an additional property. Typical i-models of restricted categories are r-combinations of category models and i-models of additional properties, such as $\left(O^{x *} A^{*}\right)^{*} B^{*}$, where $B$ is an additional property model. The rcombination $\left(O^{x *} A^{*}\right) * B^{*}$ makes the same i-model as $\left(O^{x *} B^{*}\right) * A^{*}$ when $A$ and $B$ are independent of each other. There also are cases where $A$ is a function of an additional property, such as $A^{*} x^{*}$. For example, property 'triangle' has the two variables that stand for its two interior angles. Then, a value of one of the variable, e.g., a right angle can be an additional property to 'triangle' to form 'a right angle triangle'. The property model of a right angle triangle is the r-combination of the property model of triangle and that of a right angle.

\subsection{I-models of events (event models)}

The input image of an event, 'An object $a$ has a property $P$ ', would have the pattern: The input image of $P$ with an st-value follows that of $a$ with the same st-value. This pattern relates the object model of $a$, which $O_{a}{ }^{l}$ denotes, and the property model of $P$, which $P_{m}$ denotes. That is, the input image that $O_{a}{ }^{l}$ is supposed to correspond to is followed by the input image that corresponds to $P_{m}$ and shares the st-value with the former input image. As stated in the previous section, the former input image does not directly given in general, but is given in the form of projection of the input image of $I$, which is in the PCS. This input image is denoted by $\operatorname{IM}\left(\mathrm{O}_{a}{ }^{l}\right)$.

The input image of a property $\mathrm{P}$, which follows $\mathrm{IM}\left(\mathrm{O}_{a}^{l}\right)$, is denoted by $F\left(I M\left(O_{a}^{l}\right)\right)$. Then, the relationship between $O_{a}^{l}$ and $P_{m}$ is

$\mathrm{C}_{\mathrm{t}}\left(\mathrm{F}\left(\mathrm{IM}\left(O_{a}^{l}\right)\right)\right)=P_{m}$.

As stated earlier, $\mathrm{C}_{\mathrm{t}}$, an operation to form property models from 
input images, functions as categorization of input images. The object (or its input image) that corresponds to $O_{a}{ }^{l}$ is called the object (or the input image) designated by $O_{a}{ }^{l}$. Then, this formula is paraphrased as 'the object designated by $O_{a}{ }^{l}$ has a property that corresponds to $P_{m}$.' The combination of $O_{a}{ }^{l}$ and $P_{m}$ in this relationship constitutes the event model.

This relationship determines the way $O_{a}{ }^{l}$ and $P_{m}$ correspond to the input image of the event. That is, the event model takes mode + $($ or -$)$ to an input image $d$ iff $\operatorname{Mode}\left(P_{m} \mid d\right)=+($ or -$)$, where $d$ follows the input image $d^{\prime}$ designated by $O_{a}{ }^{l}$, i.e., $\operatorname{Mode}\left(O_{a}{ }^{l} \mid d^{\prime}\right)=$ + . This combination of $O_{a}{ }^{l}$ and $P_{m}$ is called a $c$-combination and is denoted by $\left\langle O_{a}{ }^{l} \mid P_{m}\right\rangle$, where $O_{a}{ }^{l}$ is called a core and $P_{m}$ is called a subordinate.

I-models of events about properties are also c-combinations. When a property (e.g., burning) has a property (e.g., generating heat), the relationship between the i-models of these two properties is the same as that between $O^{l}{ }_{a}$ and $P_{m}$ in $\left\langle O_{a}{ }^{l} \mid P_{m}\right\rangle$, though the input image of the core is given in this case.

As well as object models or property models, the correspondence of the event models and the events is also conjecture; the information embodied by the event model is uncertain. Hence, succeeding inputs will reinforce or correct them.

The overall relationships between Agent, the environment around Agent, and the $\mathrm{i}-\mathrm{model}$ of the environment $W$ are as follows. $W$ includes $I$ and object models at certain st-values in $W$. These st-values correspond to the real agent's st-locations and objects'st-locations in the environment. Then, the relationship between object models and $I$ synchronizes with the relationship between the objects and Agent in the real world. Therefore, the correspondence between objects, which include Agent, in the world and the object models in $W$ is approximately homomorphic. This homomorphism would enable Agent to control its own body to 
interact effectively with the objects in the environment.

\section{4 I-models of quantities}

There are events about every member in a category such as 'Every bird has a vertebra', or events about the members in a subset of a category, such as 'Some birds do not fly' or 'Half of human beings are male'. In these examples, 'some birds' or 'half of human beings' are not an arbitrary some birds or half of human beings but certain birds or certain human beings, respectively.

To form i-models of these events, the quantities of the subsets of the categories must be compared with the quantities of the categories. The quantity of a category is a property of the category. When the i-model of a quantity is the subordinate of a ccombination, its core is a category model and designates not category members but the category as a unit. This is because, according to the formation of a c-combination, the subordinate property model of a c-combination conditions the designation of objects by the core. For instance, 'dangerous' in the event 'The tiger is dangerous' is a property applied to every member of the category of the tiger, and 'being in danger of extinction' in the event 'The tiger is in danger of extinction' is not a property of members of the category but a property of the category,.

The ratio of the quantity of a part of a category to the quantity of the category is a relationship between them, or a property of the ordered pair of them. Accordingly, when perceiving this property, the object that corresponds to the core $\mathrm{i}-\mathrm{model}$ is the ordered pair of the category and its part. Let $A$ be a category model, and let $B$ be a property model. Let $R$ be an i-model of the ratio of a part of a category to the category. Let the PCS perceive an event that the ratio of the part designated by $A^{*} B *$ to the category designated by $A$ corresponds to an i-model $Q$. Then, $\langle A, A * B *>$ designates the ordered pair of the category and the part via the input image from 
them. Under this designation, $Q$ corresponds to the input image of the ratio between them. Thus, the i-model of this event is $<<A, A * B *>|Q\rangle$.

Component $Y$ in $\langle X, Y\rangle-Q$ is denoted by $Q_{y}(X)$. Then, under the perception that forms $<<A, A * B *>|Q\rangle$, the PCS can form $\left\langle Q_{A * B}(A) \mid A * B *\right\rangle$

and

$\left\langle Q_{A * B *}(A) \mid B\right\rangle$.

The things designated by $Q_{A} *^{*}(A)$ is not the category but any member of the category because $B$ is the i-model of a property of members, not that of a property of a category as a unit. Hence, $A * B *$ and $B$ can be the subordinates of these event models respectively.

Let $A l$ denote the i-model of the ratio, $1: 1$. Then, $\left\langle A l_{A * B}(A) \mid A * B *\right\rangle$ is the same as $\langle A \mid A * B *\rangle$ and $\langle A \mid B\rangle$. Let $E i$ denote the i-model of the ratio other than $0: 1$. Then, the PCS can form

$\left\langle E i_{A} * B *(A)\right| A * B *>$

and

$<E i_{A * B} *(A) \mid B>$.

Let BIRD, HUMAN, MALE, FLY $Y^{N}$, and $H A L F$ be the i-models of the categories 'bird', 'human being', 'male', and properties 'not fly', and 'half' respectively. Then, the i-model of 'some birds'that has the feature 'not fly' implicitly, is $E i_{B I R D * F L Y}{ }^{*}(B I R D)$. When there exists things designated by this i-model, the PCS can form the event model of the event: Some birds do not fly,

$$
<E i_{B I R D * F L Y^{N} *(B I R D) \mid F L Y^{N}>,}
$$

i.e., $\left\langle\left\langle B I R D, B I R D * F L Y^{N *}\right\rangle \mid E i\right\rangle$.

The i-model of 'half of human beings', which includes 'male' implicitly, is $H A L F_{H U M A N^{*} M A L E *}(H U M A N)$. When there exists things designated by this i-model, the PCS can form the event model of the event: Half of human being are male, 
$<H A L F_{H U M A N * M A L E}(H U M A N) \mid M A L E>$, i.e. $\langle<H U M A N, H U M A N * M A L E *>\mid H A L F\rangle$.

\subsection{Top-down processing}

Basically, the PCS forms i-models from input images, namely, in a bottom-up fashion. These i-models should be available as acquired units to analyze new inputs efficiently. Furthermore, the PCS should have a faculty to make combination of acquired imodels to form c-combinations or r-combinations without inputs from the environment. This faculty would be necessary to guess the future or unknowns. To make combinations of i-models, the faculty should include the operation that makes the i-models take mode + without inputs. I-models formed in such a top-down fashion are extension of bottom-up i-models. I-models when they take mode + can designate their counterparts in the world. They are used to form prospective event models (hypotheses), which restrict or control the direction to look for inputs from which the PCS could acquire necessary information. These top-down i-models lack correspondence with the environment. That is, they have no basis in $W$. Thus, when the reality of top down i-models comes into question, they need to be examined whether there exist events in the environment that correspond to them.

Bottom-up event models are characterized by their correspondence to the input images of objects or events, etc. Thus, they are situated in $W$. A top-down object model, that is independent of $W$ is denoted by $\left[O^{l}\right]$. The $\mathrm{i}$-model of the existence of an object or the input image of the object is denoted by $E$. When there exists a set of $\operatorname{IM}\left(\left[O^{l}\right]\right)$, E can be formed from the set by $\mathrm{C}_{\mathrm{t}}$. Under the top down condition Mode $\left(\left[O^{l}\right]\right)=+$, when designation by $\left[O^{l}\right]$ (i.e. $\left.\operatorname{IM}\left(\left[O^{l}\right]\right)\right)$ is found, $\mathrm{C}_{\mathrm{t}}\left(\operatorname{IM}\left(\left[O^{l}\right]\right)=E\right.$. That is, $\left\langle\left[O^{l}\right] \mid E\right\rangle$ is formed. When $\operatorname{IM}\left(\left[O^{l}\right]\right)$ is not found, $\left\langle\left[O^{l}\right] \mid E^{N}\right\rangle$ is formed.

The i-models of existence of top down event models are 
formed in the same way. Let $A$ be an event model. Under the top down condition $\operatorname{Mode}([A])=+$ (the designation of the events by $[A]),\langle[A] \mid E\rangle$ or $\left\langle[A] \mid E^{N}\right\rangle$ is formed according to the existence of $\operatorname{IM}([A])$. There is a case where the designation of the core of a topdown event model $[A]$ does not exist (i.e. there is no object counterpart in the world of the core). Then, since there is no input image that $\mathrm{C}_{\mathrm{t}}$ should operate, the subordinate of [A] takes no mode. Accordingly, $[A]$ takes no mode, hence there exists no $\operatorname{IM}([A])$, and $<[A] \mid E^{N}>$ is formed.

There can be an i-model that designates a top down event model $[A]$ in the perception of an event, that is, [A] that appears in the correspondence or non-correspondence relationship to the input image of the event. This i-model is denoted by $[A]^{M} . \operatorname{IM}\left([A]^{M}\right)$ is ' $[A]$ in the perception of an event'. Thus, $\mathrm{F}\left(\operatorname{IM}\left([A]^{M}\right)\right)=$ Mode([A]). The i-model of this correspondence or mode + of $[A]$ in the perception of an event is denoted by $T$.

Then, when Mode $\left([A]^{M}\right)=+$, i.e., the designation of [A] in the perception of an event is possible,

(1) if $\operatorname{Mode}([A])=+$, then, $\mathrm{C}_{\mathrm{t}}(\operatorname{Mode}([A]))=T$ and $\left\langle[A]^{M} \mid T\right\rangle$ is formed.

(2) if $\operatorname{Mode}([A])=-$, then, $\mathrm{C}_{\mathrm{t}}(\operatorname{Mode}([A]))=T^{N}$ and $\left\langle[A]^{M}\right| T^{N}>$ is formed.

(3) if Mode([A]) takes no value, then, $C_{t}(\operatorname{Mode}([A]))$ does not work or takes no value. This is the case where the designation of the core of $A$ does not exist; accordingly, no event model is formed.

Naturally, $<[A]^{M} \mid T>$ follows $A$ as the i-model of this perception $A$ : I-model [A] corresponds to the input image of an event in the world. Thus, $E$ and $T$ are i-models of different aspects of the same perception. $E$ is the subordinate of the core [A] that designates an event, and form the i-model of existence of the event. On the other hand, $T$ is a subordinate of the core $[A]^{M}$ that designates $[A]$ in the perception of the event, and forms the i- 
model of the correspondence of $[A]$ to an event, namely truth of [A].

\section{Correspondence between i-models and linguistic expressions}

\subsection{Grammar}

In this section I examine correspondence between i-models and some grammatical structures, a problem raised in the introduction. Then, propositional logic should result from the PC about the imodel structures that are the i-model counterparts of the logical connectives.

- The subject-predicate structure represents events. Event models are constructed to correspond to the events that the declarative sentences stated in Section 2.3 represent. That is, the core-subordinate in c-combinations is the i-model counterpart of the subject-predicate in declarative sentences of the type: An object (or a category) $a$ has a property $P$. On one hand, the subject-predicate structure represents an event in which the object represented by the subject has the property represented by the predicate. On the other hand, the c-combination of the event model corresponds to the event in which the object that corresponds to, or designated by the core is followed by the property of the object that corresponds to the subordinate. Then, when the linguistic counterpart of an event model $A$ is a declarative sentence, $[A]$, which lacks the correspondence with the world, would be the imodel counterpart of 'that clause of the sentence', which is independent of reality. Moreover, when the PCS forms A, the PCS can also form $\left\langle[A]^{\mathrm{M}} \mid T\right\rangle$. Accordingly, the linguistic counterpart of $<[A]^{\mathrm{M}} \mid T>$ becomes 'that clause of the sentence, is true'.

- A typical restrictive modification such as 'a red flower' or 'a flying bird' can represent a category restricted by a property that 
corresponds to the r-combination of the category model and the property model e.g., (the i-model of a flower)*(the i-model of red)*.

- Quantifiers: 'every' and 'some' represent ratios that correspond to i-models $A l$, and $E i$, respectively.

- Disjunction of two sentences $\alpha$ and $\beta$ : $\alpha v \beta$.

Let $\{[A],[B]\}$ be the set of event models $[A]$ and $[B]$, which takes Mode $+\operatorname{iff}[A]$ and $[B]$ both take Mode + . When some members of $\{[A],[B]\}$ correspond to an event(s), then, PCS can form $<\{[A],[B]\}^{M},\{[A],[B]\}^{M *} T^{*}>\mid E i>$.

$\{[A],[B]\}^{M}$ in this core designates the $\mathrm{i}-\operatorname{model}\{[A],[B]\}$ in the $\mathrm{PC}$.

When $[A]$ and $[B]$ are the i-model counterparts of the that clauses of the sentences $\alpha$ and $\beta$, respectively, then this event model is the i-model counterpart of the disjunction $\alpha \vee \beta$.

- Conjunction: $\alpha \wedge \beta$

In the same way as disjunction, $<\{[A],[B]\}^{M},\{[A],[B]\}^{M *} T^{*},>\mid A l>$, is the $\mathrm{i}$-model counterpart of the conjunction $\alpha \wedge \beta$. This is identical with $<\{[A],[B]\}^{M} \mid T>$,

- Implication: $\alpha \rightarrow \beta$

The event represented by this sentence is that when the event represented by that clause of $\alpha$ exists (is true), an event represented by that clause of $\beta$ exists (is true). The event model of this event is $\langle[A] \mid[B]\rangle$. That is, the event that corresponds to $[B]$ follows the event that corresponds to $[A]$.

- Negation: $\neg \alpha$

This sentence represents the event that the object represented by the subject of $\alpha$ does not have the property represented by the predicate of $\alpha$. The event model of this event is $\left\langle[A]^{M} \mid T^{N}\right\rangle$. This event model can be formed when an object designated by the core 
of $A$ exists.

\subsection{Propositional logic}

The basis of propositional logic is the logical connectives as truth-value functions and the inference rule, modus ponens: A proposition expressed by a sentence $\beta$ is inferred from the propositions expressed by the sentences $\alpha$ and $\alpha \rightarrow \beta$. I-model counterparts of disjunction, conjunction, and negation stated in Section 3.1 satisfy the truth conditions of the logical connectives as truth-value functions. For instance, when the mode of $[A]$ or $[B]$ is + , the i-model counterpart of $\alpha v \beta$ takes mode + . Then, <(the imodel counterpart of $\alpha \vee \beta)^{M} \mid T>$ is formed. When both of their modes are - , the i-model counterpart of $\alpha v \beta$ takes mode -, resulting in formation of <(the i-model counterpart of $\alpha v \beta)^{M} \mid T^{N}>$. Only the i-model counterpart of implication lacks the i-model counterpart of the truth condition in the case where the presupposition is false: When $\alpha$ is false, $\alpha \rightarrow \beta$ is true regardless of whether $\beta$ is true or false. In this case, no event corresponds to $[A]$. Therefore, the event model $\langle[A]|[B]>$ takes no mode, or no imodel is formed. This corresponds to a situation in which this sentence has no information. Accordingly, this case can be eliminated.

The i-model counterpart of modus ponens is formed according to the following steps.

(1) I-models $[A]$ and $[\langle[A] \mid[B]\rangle]$ are set up and combined in a topdown manner to construct the i-model counterpart of "that $\alpha$ and $\alpha$ $\rightarrow \beta^{\prime}:$

$\left[<\left\{[A]^{M},[<[A] \mid[B]>]\right\}^{M} \mid T>\right]$.

(2) This is the i-model of an event consisting of two events, $e_{1}, e_{2}$ that correspond to $[A]$ and $[\langle[A] \mid[B]\rangle]$, respectively. $[\langle[A] \mid[B]\rangle]$ corresponds to any $e_{2}$ that satisfies the condition: [B] corresponds to a part of $e_{2}$ under the correspondence of [A] to the other part of 
$e_{2}$. Then, since the latter part of $e_{2}$ is identical with $e_{1}$, which corresponds to $[A]$, the condition for $[B]$ to correspond to the former part of $e_{2}$ is satisfied. Thus, Steps (1) and (2) forms the imodel counterpart of modus ponens, $<\left[<\{[A],[<[A] \mid[B]>]\}^{M}|\mathrm{~T}\rangle\right] \mid[B]>$.

Thus, the PCS can hold propositional logic.

\subsection{The liar paradox}

As is well known, the liar sentence, 'This sentence is false' causes a paradox: If the liar sentence is true (false), the liar sentence becomes false (true) as a logical consequence. Thus, The liar paradox causes a problem of the truth of the information expressed by the liar sentence. If i-models embody pieces of information that linguistic expressions express, then the i-model counterpart of the liar sentence would reveal what information the liar sentence expresses, why the paradox occurs, and how the paradox can be avoided.

To tackle this problem, a preparation is necessary. $T$, the imodel counterpart of 'truth', is the i-model used to evaluate whether an event model in question corresponds to a certain event. This truth evaluation ( $T$ evaluation) of an event model is PC of the result of PC. Therefore, results of $T$ evaluation can also be objects of $T$ evaluation. However, superposition of $T$ evaluation of an event model is equivalent to no $T$ evaluation of the perception of the event. That is, because the information of an event does not need $T$ evaluation, superposition of $T$ evaluation can be reduced to the basic two cases. Let $A$ be an event model. Then, 1. (the PCS can form) $\left\langle\left[\left\langle[A]^{M} \mid T\right\rangle\right]^{M} \mid T\right\rangle$ iff (the PCS can form) $<[A]^{M} \mid T>$ iff (the PCS can form) $A$.

The sentence whose i-model counterpart is $A$ is denoted by $S(A)$. Then, the above is the i-model counterpart of "that that $\mathrm{S}(A)$ is true is true iff that $\mathrm{S}(A)$ is true iff $\mathrm{S}(A)$ '. 
2. In the same way as the above, $<\left[<[A]^{M}\left|T^{N}\right\rangle\right]^{M}\left|T^{N}\right\rangle$ iff $\left\langle[A]^{M} \mid T^{N N}\right\rangle$ iff $\left\langle[A]^{M} \mid T\right\rangle$ iff $A$.

3. $<\left[<[A]^{M} \mid T>\right]^{M} \mid T^{N}>$ iff $\left\langle[A]^{M}\right| T^{N}>$ iff $A^{N}$,

4. $<\left[<[A]^{M} \mid T^{N}>\right]^{M} \mid T>$ iff $\left\langle[A]^{M}\right| T^{N}>$ iff $A^{N}$.

The liar paradox can now be examined in the following way.

The original liar sentence is

$L:$ This sentence is false.

A function from a demonstrative $x$ to the sentence that satisfies $x$ is denoted by $\operatorname{SENTENCE}(x)$. Then, 'This sentence' in $L$ is replaced with SENTENCE(this), whose value is $L$, and $L$ is 'SENTENCE(this) is false.' Let $\mathrm{S}^{-1}(L)$ be the i-model counterpart of $L$. Then,

$\mathrm{S}^{-1}(L)=<\left[\mathrm{S}^{-1}(\operatorname{SENTENCE}(\text { this }))\right]^{M}\left|T^{N}\right\rangle$.

Substitution of $L$ for SENTENCE(this) in the right side of this equation and application of this equation to the obtained result is $\mathrm{S}^{-1}(L)=\left\langle\left[\left\langle\left[\mathrm{S}^{-1}(\operatorname{SENTENCE}(\text { this }))\right]^{M} \mid T^{N}\right\rangle\right]^{M} \mid T^{N}\right\rangle$

The right side of Formula (2) is reduced to

$<\left[\mathrm{S}^{-1}(\operatorname{SENTENCE}(\text { this }))\right]^{M}|T\rangle$.

This contradicts Formula (1). Repeats of this process cause alternate appearances of $T$ and $T^{N}$ of $\left[\mathrm{S}^{-1}(L)\right]^{M}$. This is the i-model counterpart of the liar paradox.

However, is this construction of the i-models possible? In fact, this construction has two difficulties. First, the substitution to determine SENTENCE(this) gives itself as its value. This does not provide the i-model for $T$ evaluation. Second, both $T^{N} \mathrm{~s}$ in Formula (2) are subordinates in the same event model $\mathrm{S}^{-1}(L)$. That is, the $T$ evaluation used to form the i-model represented by this formula operates on itself, $\mathrm{S}^{-1}(L)$. This is impossible because $T$ evaluation cannot operate on itself. $\mathrm{S}^{-1}(L)$ is not within the scope of the identical $T$ evaluation in $S^{-1}(L)$. Because of these difficulties, $\mathrm{S}^{-1}(L)$ cannot be constructed as an event model. That is, the liar sentence has no counterpart of an event model, or no information 
in the form of an i-model.

In the same way,

$C$ : This sentence is true,

also has no counterpart of an event model. In contrast,

$D$ : This sentence is written in English.

has a similar self-reference structure but is true. 'This sentence' in $D$ designates sentence $D$ as a sequence of English words, not as a sentence that expresses a piece of information. Thus, SENTENCE(this) in D is fixed, and $S^{-1}(D)$ lies within the scope of the $T$ evaluation.

This perception, 'to be beyond or within the scope of $T$ evaluation', is also evaluation about application of $T$ evaluation. The i-model of this evaluation is denoted by $T^{2}$. When $\mathrm{S}^{-1}$ (a sentence) is within the scope of $T$ evaluation, then $<\left[\mathrm{S}^{-1}(\text { a sentence })\right]^{M} \mid T^{2}>$

is formed. Therefore, $<\left[\mathrm{S}^{-1}(L)\right]^{M} \mid T^{2 N}>$ and $\left\langle\left[\mathrm{S}^{-1}(C)\right]^{M} \mid T^{2 N}\right\rangle$, but $\left\langle\left[\mathrm{S}^{-1}(D)\right]^{M} \mid T^{2}\right\rangle$ are formed in the PCS.

Tarski's levels of languages, namely, language, meta-language, meta-meta-language, etc., (A.Tarski, 1983, Chapter 8 pp152-278) differentiate between $T$ evaluation and objects of the $T$ evaluation. Thus, the levels avoid the second difficulty but leave the first one is left untouched.

Kripke's 'truth value gaps', being unable to fix the truth value of the liar sentence because of the first difficulty (Kripke. S, 1975.), is similar to but different from being unable to fix the object of $T$ evaluation. Both approaches reach similar results regarding the liar sentence: " $S^{-1}(L)$ does not fix the object of $T$ evaluation' vs. ' $L$ does not have a specific truth value'. However, the two approaches reach different results regarding $C$ : ' $\mathrm{S}^{-1}(C)$ does not fix the object of $T$ evaluation' vs. 'The truth value of $\mathrm{C}$ is arbitrary' and ' $\mathrm{S}^{-1}(\neg C \vee C)$ does not fix the object of $T$ evaluation' 
vs. ' $\neg C \vee C$ is true'.

The sentences that express $T^{2}$ evaluation with the same structure as those of $L$ or $C$ cause the same problems that $L$ and $C$ do. Let's examine the following sentences.

$G$ : This sentence is beyond $T$ evaluation. Then, $\mathrm{S}^{-1}(G)=\left\langle\left[\mathrm{S}^{-1}(\operatorname{SENTENCE}(\text { this }))\right]^{M} \mid T^{2 N}\right\rangle$.

Because of the first difficulty stated above, 'This sentence' in $G$ is beyond $T$ evaluation. Hence, $G$ is true. This is a $T$ evaluation. That is, $G$ is within $T$ evaluation, thus falling into a contradiction.

In the same way, the sentence,

$H$ : This sentence is within $T$ evaluation,

whose i-model counterpart is

$\mathrm{S}^{-1}(H)=\left\langle\left[\mathrm{S}^{-1}(\operatorname{SENTENCE}(\text { this }))\right]^{M} \mid T^{2}\right\rangle$,

also causes a contradiction. Since 'This sentence' in $H$ is beyond $T$ evaluation, $H$ is false, hence is within $T$ evaluation. At the same time, because of falsity of $H, H$ is beyond $T$ evaluation.

$G$ and $H$ have the same structure as $L$ and $C$ respectively, where $T^{2}$ evaluation in their subordinates operates on itself in the cores.

Thus, $G$ and $H$ are beyond $T^{2}$ evaluation and the i-model counterparts of $G$ and $H$ have no information. Thus, $T^{2}$ evaluation also causes the same matters as those by $T$ evaluation. Accordingly, a higher level of evaluation is necessary to perceive these matters. This increase in evaluation levels is infinite. Although sentences such as $L, C, G, H$ can be composed on these levels, i-model counterparts of these sentences have no information. That is, these sentences express or convey no information in the form of imodels.

\section{Discussion and conclusion.}

In this article, I tried to found grammatical structures of 
linguistic expressions and propositional logic on the human perceptual-cognitive system, the PCS. The basis of this study is the idea that the PCS acquires information of events from the inputs sent from the environment, and the information should be embodied in some physical entities in the PCS that should have some structures that correspond to structures of events. This idea leads to the conjecture that since linguistic expressions express the items of the information about events, certain structures of the physical entities will be the perceptual-cognitive foundations of the grammatical structures of the linguistic expressions. I worked on this problem within the scope of the computational theory of the PCS.

As a result, c-combination was acquired as the i-model counterpart of the subject-predicate structure of declarative sentences, and some i-model structures that correspond to restrictive modification and logical connectives were acquired. Naturally, propositional logic results from these structures. The result was also applied to the liar paradox, resulting in a new solution. These demonstrations support the possibility that imodels with these structures exist and are perceptual-cognitive foundations of those grammatical structures.

This strategy to found grammatical structures on i-model structures may be applied to semantics, the study of the meanings expressed by linguistic expressions. This is because the meaning of linguistic expressions can be regarded as the information expressed by the linguistic expressions on the basis of the following properties of meaning, (1), the PCS forms primitive or elemental meanings from accumulation of experiences or input from the world, (2), the PCS memorizes the meanings, (3), the PCS represents events using linguistic expressions according to their meanings, 
(4), For the PCS, to understand the meanings of linguistic expressions is to acquire the information expressed and conveyed by the linguistic expressions.

Identification of meaning with information and the results of this article implies that i-models are perceptual-cognitive foundations of meanings. Then, compositionality of meanings is supported within the range of the correspondence between i-model structures and those of grammatical structures.

I-models with these structures is a hypothesis. For the confirmation of this hypothesis, they must be consistent with physiological evidence in addition to linguistic evidence. The basic problems are the confirmation of component i-models: object models, property models, category models, and the formation of these i-models from input. When these $\mathrm{i}-$ models are found in the PCS, it would be possible in principle to examine those i-model structures in neurophysiology. C-combination determines the way in which event models correspond to inputs, given the correspondence of their component i-models.

For instance, this hypothesis predicts the following: When a dog named Ben is brown, PC of this event by the PCS is formation of the c-combination of the property model of brown and the object model of Ben. Therefore, the following three conditions are necessary to confirm this prediction.

(1). There exist two i-models corresponding to Ben and brown: $\mathrm{S}^{-1}($ Ben $)$ and $\mathrm{S}^{-1}($ brown).

(2). There is a synthesis composed of these two i-models that takes mode + iff under the designation of Ben by $\mathrm{S}^{-1}($ Ben $), \mathrm{S}^{-1}$ (brown) sharing the common st-value with $\mathrm{S}^{-1}(B e n)$ ' takes mode + . (3). After this event model is formed, when the $\mathrm{S}^{-1}$ (Ben) takes Mode + , the $\mathrm{S}^{-1}($ brown) in the event model takes mode + without an input.

This hypothesis about i-model structures depends on the 
computational theory of the PCS and event structures, which does not include a condition specific to human beings. Therefore, the hypothesis could apply to any living organisms that have a perceptual-cognitive system with a capacity to identify or discriminate a various kinds of objects and properties. Such living organisms will show behavior following guesses based on their experience. This is also a prediction of this hypothesis.

\section{Reference}

Anderson, J. (1990). The adaptive nature of thought.

Hillsdale, NJ: Erlbaum.

Geisler, W. \& Diel, R. (2003). A Bayesian approach to the evolution of perceptual and cognitive systems. Cognitive Science, $27,379-402$.

Jackendoff, R. (1983). Semantics and Cognition. Cambridge. MIT press.

Jackendoff, R. (2002). Foundation of language: Brain, Meaning, Grammar, Evolution. Oxford university press.

King, J. C. (2018). Propositions. In Michael G Lanzberg (Ed), The Oxford Handbook of Truth Part IV Propositions and Truth bearers (pp. 1-42). Oxford university press.

King, J. C. (2019). On propositions and Fineness of Grain. Synthese 196(4), 1343-1367. 
Kripke, S. (1975). An Outline of a Theory of Truth. Journal of Philosophy 72, 690-716.

Langacker, R. (1987). Foundation of cognitive grammar. Vol. 1. Stanford university press.

Langacker, R. (2008). Cognitive grammar: a basic introduction. Oxford university press.

Marr, D. (1982). Vision. New York: W. H. Freeman.

Soames, S. (2018). Cognitive propositions in realist linguistics. In Christina Behme and Martin Neef (Eds), Essays on Linguistic Realism (Chapter 9, PP 235-252). John Benjamines :NL.

Talski, A. (1956). The concept of truth in formalized languages. In his Logic, Semantics, Metamathematics (Chapter 8, pp. 152-278). Oxford university press. 